\title{
Remoción de Cromo Hexavalente de Soluciones Acuosas usando Cáscara de yuca (Manihot esculenta): Experimentos en Columna
}

\section{Removal of Hexavalent Chromium from Aqueous Solution using Cassava Peel (Manihot esculenta): Column Experiments}

\author{
DOI: http://dx.doi.org/10.17981/ingecuc.13.1.2017.04
}

Artículo de investigación. Fecha de Recepción: 24 de Noviembre de 2016. Fecha de Aceptación: 27 de diciembre de 2016

\author{
Alberto Ricardo Albis Arrieta \\ Universidad del Atlántico. Barranquilla (Colombia) \\ albertoalbis@mail.uniatlántico.edu.co \\ Jose Daniel Ortiz Toro \\ Naturmega S.A. Barranquilla (Colombia) \\ jdortiz@naturmega.com.co \\ Jader Enrique Martínez De La Rosa \\ Universidad del Atlántico. Barranquilla (Colombia) \\ jaderemartinez@mail.uniatlantico.edu.co
}

Para citar este artículo:

A. M. Orjuela Garzón, J. E. Colmenares Montañez, and L. Viveros Rosero, "Remoción de cromo hexavalente de soluciones acuosas usando cáscara de yuca (Manihot Esculenta): experimentos en columna”, INGE CUC, vol. 13, no. 1, pp. 42-52, 2017. DOI: http://dx.doi. org/10.17981/ingecuc.13.1.2017.04

\section{Resumen}

Introducción- El cromo hexavalente es un metal altamente tóxico y es considerado como uno de los contaminantes de mayor impacto ambiental. El uso de bioadsorbentes se ha considerado como una alternativa viable para su remoción, por lo que es necesario estudiar el comportamiento de estos adsorbentes en operaciones unitarias semejantes a las empleadas en la industria.

Objetivo- Estudiar el potencial de la cáscara de yuca como biosorbente en la adsorción de cromo hexavalente en un proceso semicontinuo en columna de lecho empacado.

Metodología-Se analizó la influencia del caudal de alimentación de soluciones de cromo, la concentración de dicho caudal y la altura del lecho sobre la capacidad de remoción y el tiempo de ruptura en un montaje a escala de laboratorio.

Resultados- Hay un mayor rendimiento cuando se utilizan el flujo de alimentación y la concentración inicial más bajos, así como la mayor altura del lecho. El comportamiento dinámico fue ajustado a tres modelos clásico de experimentos en columna. No obstante, el mejor ajuste se alcanzó con el modelo estadístico de superficie de respuesta.

Conclusiones- La concentración inicial de cromo en la corriente de alimento es el factor que tiene mayor efecto sobre la capacidad de remoción y el tiempo de ruptura en columnas de lecho fijo empacadas con cáscara de yuca.

Palabras clave- Cáscara de yuca, cromo, remoción, tiempo de ruptura, columna empacada

\section{Abstract}

Introduction- Hexavalent chromium is a highly toxic metal and it is considered one of the contaminants with the highest environmental impact. Bioadsorbents have been considered as a viable option for chromium removal, leading to the necessity to study the behavior of these adsorbents in unit operations similar to the ones employed in the industry.

Objective- To study the potential of cassava peels as bio-sorbent in the adsorption of hexavalent chromium in a semi-continuous process in fixed bed columns.

Methodology- The effect of parameters such as feed flow rate, initial concentration and bed column height on the response variables rupture time and removal capacity was analyzed using a laboratory scale experimenta set-up.

Results- Low feed flow rates, low initial concentrations, and higher bed height produce the best conditions for chromium removal. Additionally, three classical models for removal on packed column were used to fit the dynamic behavior of this process and to obtain significantly physical parameters. However, the best fitting was obtained using the model of the response surface.

Conclusions- The concentration of chromium in the feed stream is the experimental factor that has the most influence on removal capacity and rupture time in fixed bed columns packed with cassava peel.

Keywords- Cassava peel, Chromium, Removal, Rupture time, Packed Column. 


\section{Introducción}

La contaminación de los cuerpos de agua por metales pesados es uno de los mayores problemas ambientales en la actualidad. Metales como el plomo [1], cobre [2], cadmio [3, 4], zinc [5], mercurio [6], níquel [7] y cromo [8] provenientes de diferentes actividades industriales como la minería, la industria de pinturas, la fabricación de baterías, los recubrimientos, las impresiones, la industria energética, los fertilizantes, los plaguicidas y los textiles tienen efectos negativos sobre la salud humana (anemia, anorexia, malestar, pérdida de apetito, daño cerebral, de hígado, de riñón, daño gastrointestinal y retraso mental en los niños) y el ambiente. La mayoría de los iones metálicos son tóxicos para los seres vivos. Estos iones metálicos no son degradables y son persistentes en el entorno. Además, la eliminación de estos de las aguas residuales es importante para proteger la salud pública. Los efluentes industriales son una de las principales causas del aumento de la concentración de los metales pesados. Este aumento se debe a procesos que involucran corrosión de tuberías de agua, residuos de vertido, galvanoplastia, electrólisis, electro-ósmosis, minería, procesos relacionados con energía y combustibles, fertilizantes, pesticidas, hierro y acero, procesos en curtiembres, tratamiento de superficies metálicas, procesos fotográficos, residuos de instalaciones aeroespaciales y de energía atómica, etc. [9]. Por lo tanto, la eliminación y recuperación de metales pesados de corrientes de efluentes son esenciales para la protección del medio ambiente [10].

Todos los metales pesados se encuentran presentes en medios acuosos (el agua químicamente pura no existe en la naturaleza), aunque sus concentraciones en los cuerpos de agua naturales son muy bajas. En particular, el cromo hexavalente es reconocido como un metal altamente tóxico y considerado como uno de los contaminantes de mayor impacto. El cromo es comúnmente liberado a cuerpos de agua naturales como descarga de una amplia variedad de aguas residuales procedentes de la actividad industrial. Por otro lado, catalizadores que emplean cromo son ampliamente utilizados en diversidad de procesos químicos que incluyen la oxidación selectiva de hidrocarburos. De acuerdo con los lineamientos de la Organización Mundial de la Salud (OMS), la concentración máxima permisible de cromo en aguas potables es de $0.05 \mathrm{mg} / \mathrm{L}$ [11].

Una serie de tecnologías para la eliminación de contaminantes inorgánicos y orgánicos de soluciones acuosas se han desarrollado a lo largo de los años, incluyendo el intercambio iónico [12], adsorción [13], precipitación [14], degradación fotocatalítica [15], filtración por membrana [16] y los métodos electroquímicos [17]. De éstos, la adsorción es considerada como la que tiene más potencial debido a su alta eficiencia y fuerte capacidad para eliminar contaminantes [18]. La adsorción es un proceso de tratamiento simple que requiere menos inversión y no crea contaminación secundaria de lodos biológicos [19]. Las nuevas prácticas se han centrado en la búsqueda de adsorbentes eficientes, que incluso, utilizan métodos complejos con adsorbentes que consisten en nanotubos de carbono magnéticos multicapa [20] y adsorción sobre nanopartículas de hierro [21].

Métodos alternativos de recuperación o remoción de metales que se basan en las propiedades de adsorción de ciertas materias primas de origen natural están siendo considerados [22]. Tomando como criterios el bajo costo y la capacidad de adsorción de los residuos agrícolas, un proceso que ha resultado promisorio para la remoción de metales pesados como $\mathrm{Cd}(\mathrm{II}), \mathrm{Pb}(\mathrm{II})$, y $\mathrm{Cr}$ (III), es la biosorción con un material de bajo costo y de fácil acceso como es la cáscara de yuca (Manihot esculenta) [23]; sin embargo, estos estudios de adsorción en disolución se han realizado mediante experimentos en lotes que, aunque son de buena eficiencia, presentan las desventajas de cualquier proceso discontinuo. Una alternativa atractiva para superar este problema es usar columnas de flujo continuo de lecho adsorbente fijo. El uso de una columna de adsorción hace que el proceso sea continuo, de modo que sea económicamente viable a escala industrial. Después de la adsorción de iones de metales los adsorbentes se pueden regenerar con éxito usando ácidos diluidos. Estas columnas se pueden utilizar entonces repetidamente para la adsorción de la misma sal durante más de diez veces o de sales diferentes en sucesión sin pérdida observable en el poder de adsorción [24]. De hecho, esta es la importancia de implementar un sistema de lecho empacado para la remoción de metales pesados. Así, en esta investigación se propone el tratamiento de soluciones acuosas con cromo hexavalente basado en los estudios de adsorción en una columna de lecho fijo, utilizando la cáscara de yuca como medio adsorbente. (Volesky 1990)(Volesky 1990)(Volesky 1990) (Volesky 1990)

\section{Metodología}

\section{A. Preparación de las disoluciones de cromo}

Las disoluciones de cromo fueron preparadas usando $\mathrm{K}_{2} \mathrm{Cr}_{2} \mathrm{O}_{7}$. Se preparó una disolución patrón (1000 $\mathrm{mg} / \mathrm{L}$ ) de $\mathrm{Cr}$ mediante la adición de $2,83 \mathrm{mg} \mathrm{K}_{2} \mathrm{Cr}_{2} \mathrm{O}_{7}$ en un litro de agua destilada y desionizada. A partir de ella se prepararon las disoluciones necesarias para cada prueba y luego se ajustaba el $\mathrm{pH}$ de cada disolución agregando gota a gota una solución diluida de $\mathrm{H}_{2} \mathrm{SO}_{4}$ hasta llegar a un $\mathrm{pH}$ de $3 \pm 0.5$.

\section{B. Biosorbente}

La cáscara de yuca fue recolectada de una finca ubicada en Corozal, Sucre, la cual fue lavada con una hidrolavadora en una disolución de ácido sulfúrico a pH 3 para retirar la suciedad de la superficie de la cáscara de yuca. El secado de la cáscara de yuca se 


\section{REMOCIÓN DE CROMO HEXAVALENTE DE SOLUCIONES ACUOSAS USANDO CÁSCARA DE YUCA (MANIHOT ESCULENTA): EXPERIMENTOS EN COLUMNA}

realizó en dos etapas: en primer lugar, se secó al sol por un lapso de 12 horas y luego a $105^{\circ} \mathrm{C}$ durante 24 horas en un horno. Posteriormente, se trituró y se ajustó a un tamaño de partícula entre 250 y 400 $\mu \mathrm{m}[9]$.

\section{Montaje experimental}

Para el estudio de la biosorción se puso en marcha un montaje consistente en una columna de vidrio de $20 \mathrm{~cm}$ con lecho de cáscara de yuca pretratada y mezclada con anillos rasching de vidrio para evitar el desplazamiento del lecho, un tanque de alimento para la disolución inicial conectado a una bomba peristáltica que llevó la disolución desde la parte inferior de la columna pasando a través del lecho, donde ocurre el proceso de biosorción, y luego abandonándola en la parte superior donde fueron recolectadas las muestras a lo largo del tiempo. La Fig. 1 muestra un esquema del montaje descrito.

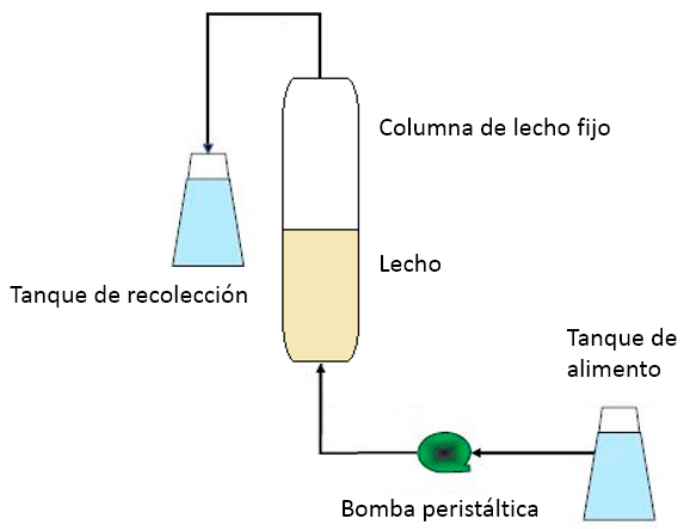

Fig. 1. Esquema del montaje de la columna de lecho empacado. Fuente: Autores.

\section{Diseño experimental}

Con base en estudios bibliográficos [25-31] se seleccionaron los parámetros de interés a estudiar y el dominio experimental para cada uno de ellos. Se consideró un sistema con tres factores, los cuales se resumen en la Tabla 2 junto con los valores altos y bajos seleccionados para el diseño factorial $2^{3}$.

TABla. 2. Factores y VAlores altos y Bajos SELECCIONADOS PARA EL DISEÑO DE EXPERIMENTOS.

\begin{tabular}{|c|c|c|}
\hline Factores & nivel bajo & nivel alto \\
\hline Caudal de alimentación (mL/min) & 4 & 10 \\
\hline $\begin{array}{c}\text { Concentración del flujo de } \\
\text { alimentación (mg/L) }\end{array}$ & 40 & 100 \\
\hline Altura del lecho $(\mathrm{cm})$ & 4,4 & 14,3 \\
\hline
\end{tabular}

Fuente: Autores
Los experimentos se realizaron en orden aleatorio para minimizar los efectos de otros factores no considerados sobre los resultados y proporcionar seguridad en la precisión de los datos. Se seleccionó el tiempo de ruptura en segundos como variables de respuesta.

\section{E. Determinación del $p H$}

Se agregaron 2.2 g de cáscara de yuca tratada en 100 $\mathrm{mL}$ de agua destilada. Posteriormente, se calentó dicha mezcla hasta alcanzar la ebullición y se mantuvo en ese punto durante $2 \mathrm{~min}$, después se realizó la filtración de la solución y con los primeros $25 \mathrm{~mL}$ se enjuagó el vaso donde cayó el filtrado; por último, se filtró el resto de la mezcla y se realizó la medición del $\mathrm{pH}$ de la solución usando un medidor de $\mathrm{pH}$ Thermo Electronic Corporation, modelo Orión 210A+ [32].

\section{F. Determinación de la humedad}

Para la determinación de la humedad se siguieron las instrucciones de la norma D3173-87. Se tomó una muestra significativa de la cáscara de yuca, la cual fue pesada y llevada a un horno a temperatura de $105^{\circ} \mathrm{C}$ por un periodo de 24 horas, terminado el tiempo se tomó la muestra y se pesó nuevamente. Se comparó el peso inicial de la muestra antes y después del secado, y el resultado de ésta dio el peso en agua que se evaporó con el calentamiento, la cual se expresó como humedad.

\section{G. Determinación de la porosidad}

La porosidad se determinó por el desplazamiento del aire en el lecho, mediante agua en un determinado volumen de yuca. En una probeta se colocó una muestra de cáscara de yuca y se enrasó con agua hasta el nivel de la muestra. Seguidamente, se estableció por diferencia entre el volumen de agua inicial y el final. La porosidad se determinó con la ecuación (1), todo esto de acuerdo con el procedimiento indicado por la Norma Europea EN-99-1.

$$
\text { porosidad }=\frac{\text { Volumen de agua empleada }}{\text { Volumen de muestra }}
$$

\section{H. Determinación de la concentración de cromo hexavalente de las soluciones}

En todas las soluciones y muestras, la concentración de $\mathrm{Cr}$ (VI) se midió usando un espectrómetro S2 PICOFOX cuyo principio es el de espectroscopia de fluorescencia de rayos X de reflexión total. Se utilizó un estándar interno de galio (Sigma-Aldrich).

\section{Densidad}

Se midió el volumen de una muestra de residuo de cáscara de yuca de masa ya conocida y se calculó la relación entre masa y volumen. 


\section{J. Análisis de espectroscopia infrarroja}

Se tomó una muestra de $0.0018 \mathrm{~g}$ de cáscara de yuca y se mezcló hasta homogenizar con $0.036 \mathrm{~g}$ de $\mathrm{KBr}$. Posteriormente, se creó una película a partir de esta mezcla y luego se analizó en un espectrofotómetro de luz infrarroja de transformada de Fourier (FT-IR Shimadzu IR Affinity-1). La temperatura a la que se realizó el análisis fue de $21^{\circ} \mathrm{C}$. El rango de frecuencia de análisis estuvo comprendido entre 400 y $4000 \mathrm{~cm}^{-1}$.

\section{K. Modelos del comportamiento dinámico}

La curva que resulta de graficar la relación de la concentración del efluente con la concentración del caudal de alimentación se conoce como curva de ruptura. El tiempo y la forma de la curva son fundamentales para analizar el comportamiento de la columna de lecho fijo determinando la cantidad de metal que puede retener el lecho, la cual se expresa usualmente como el cociente de la concentración final y la inicial en función del tiempo de contacto, la altura del lecho y el flujo. Los parámetros de la columna de lecho fijo se obtienen a partir de las ecuaciones (2) a (8).

$$
V_{e f}=Q * t_{t o t a l}
$$

Donde $\mathrm{V}_{\text {ef }}$ es el volumen del efluente $(\mathrm{mL}), \mathrm{t}_{\text {total }}$ es el tiempo total de operación [33] y Q es el caudal que circula por la columna ( $\mathrm{mL} / \mathrm{min})$.

$$
q_{\text {total }}=\frac{Q}{1000} \int_{t=0}^{t_{\text {total }}} C_{R} * d t
$$

Donde $\mathrm{C}_{\mathrm{R}}$ es la concentración de metal retenido $(\mathrm{mg} / \mathrm{L})$.

$$
\begin{aligned}
& \% R=\frac{q_{\text {total }}}{m_{\text {total }}} * 100 \\
& q_{e}=\frac{q_{\text {total }}}{W}
\end{aligned}
$$

Donde $\mathrm{q}_{\mathrm{e}}$ es la capacidad de adsorción (mg de metal adsorbido/g de biosorbente).

$$
C_{e}=\frac{m_{\text {total }}-q_{\text {total }}}{V_{\text {ef }}} * 1000
$$

Donde $\mathrm{C}_{\mathrm{e}}$ es la concentración de equilibrio del metal (mg/L).

$$
\begin{aligned}
& R_{t}=\frac{V_{c o l} * \text { Porosidad }}{Q} \\
& V_{c o l}=L * \frac{D^{2}}{4} * \pi
\end{aligned}
$$

Donde $R t$ es el tiempo de residencia en min, $Q$ es el caudal de entrada a la columna en $\mathrm{mL} / \mathrm{min}, L$ es la altura del lecho en la columna empacada en $\mathrm{cm}$, $D$ es el diámetro interno de la columna en cm y Vcol es el volumen de la columna delimitado por el lecho en $\mathrm{mL}$.
Para el posterior tratamiento de datos se utilizaron los modelos que se describen a continuación:

\section{Modelo de Thomas}

Es uno de los modelos más usados para describir curvas de adsorción en columnas continuas. Se basa en una cinética de adsorción de segundo orden y considera que la adsorción no está limitada por la reacción química, sino por la transferencia de masa entre la interfaz solido-líquido. El modelo se describe mediante la siguiente ecuación (8) [34].

$$
\frac{C}{C_{0}}=\frac{1}{1+\exp \left[\frac{k_{T H}}{Q}\left(q_{0} X-C_{0} V\right)\right]}
$$

Donde es la constante de Thomas $\left(\mathrm{ml} \mathrm{min} \mathrm{mg}^{-1} \mathrm{mg}\right.$ $\left.{ }^{1}\right)$, es la concentración máxima de soluto en la fase solida $\left(\mathrm{mgg}^{-1}\right)$ y $X$ es la cantidad de adsorbente en la columna (g). La forma lineal del modelo de Thomas es representada por la ecuación (10).

$$
\ln \left(\frac{c_{0}}{C}-1\right)=\frac{k_{T H} q_{0} m}{Q}-\frac{k_{T H} C_{0}}{1000 Q} V_{e f}
$$

Si se representa $\ln ((\mathrm{CO} / \mathrm{C})-1)$ frente $V e f$ para un caudal determinado, de la pendiente y el intercepto se obtienen los parámetros del modelo y .

\section{Modelo de Yoon y Nelson}

Este modelo asume que la velocidad de disminución de la probabilidad de la adsorción para cada molécula de adsorbato es proporcional a la probabilidad de adsorción del adsorbato y a la probabilidad de saturación en el adsorbente [34]. Es decir, a medida que avanza la adsorción, disminuye la probabilidad que ésta continúe. Para un sistema de adsorción en lecho fijo y de un solo componente, este modelo se describe a través de la ecuación (11).

$$
\ln \frac{Q}{1-Q}=k_{Y N}(\tau-t)
$$

En su forma lineal:

$$
\ln \left(\frac{c_{0}}{C}-1\right)=k_{Y N}(\tau-t)
$$

\section{N. Modelo de dosis y respuesta}

Este modelo minimiza el error de los resultados obtenidos al aplicar el modelo de Thomas, especialmente para curvas de ruptura en donde los tiempos en que se realizan los experimentos son muy largos o muy cortos [34]. El modelo se describe mediante la ecuación (13).

$$
\frac{c}{c_{0}}=1-\frac{1}{1+\left(\frac{V_{e f}}{b}\right)^{a}}
$$

Alcanzado el 50 \% del nivel de retención, la anterior ecuación puede escribirse como: 


$$
0,5=1-\frac{1}{1+\left(\frac{V_{e f(50 \%)}}{b}\right)^{a}}
$$

Según el modelo de Thomas puede determinarse que, por lo tanto, el modelo de dosis -respuesta sería representado así:

$$
\frac{C}{C_{0}}=1-\frac{1}{1+\left(\frac{C_{0} V_{e f}}{q_{0 m}}\right)^{a}}
$$

De forma lineal se tiene:

$$
\ln \left(\frac{C}{C_{0}-C}\right)=\operatorname{aln} V_{e f}-\operatorname{aln}\left(\frac{q_{0 m}}{C_{0}}\right)
$$

Los valores de y se hallan a partir de la representación de $\ln \left(C / C_{0^{-}} C\right)$ frente al volumen o al tiempo, a partir de la pendiente y la ordenada al origen.

\section{Resultados y Discusión}

\section{A. Caracterización del adsorbente}

El adsorbente objeto de estudio consistió en cáscara de yuca, que presenta una pequeña fracción de almidón como resultado inherente del proceso de selección y limpieza, con un diámetro de partícula seleccionado por tamizado que oscila en un rango de 250-500 $\mu \mathrm{m}$. Se realizaron diferentes mediciones del $\mathrm{pH}$ de la cáscara de yuca que arrojaron un promedio de 4.5, identificando al polvo usado como un biosorbente ácido. Este pH también puede ser atribuido al lavado ácido al que es sometido en el pretratamiento [35].

Mediante el procedimiento descrito en el apartado II. $F$ se obtuvo un porcentaje de humedad promedio de $10.76 \%$. Partiendo de un volumen de cáscara de yuca de $12,5 \mathrm{ml}$ se llenó hasta el tope de volumen de cáscara de yuca con $7,4 \mathrm{ml}$ de agua y usando la ecuación (1) se obtuvo una porosidad de 0.592 o $59.2 \%$. Luego, se halló la densidad con la conocida relación masa-volumen. La densidad promedio fue de $=0.41 \mathrm{~g} / \mathrm{ml}$. Este valor está de acuerdo con los resultados reportados por Wilaipon, según el cual, la densidad de la cáscara oscila en un rango de 0.40-0.77g/ml [36]. En general, los resultados obtenidos en la caracterización son inherentes de este tipo de biomasas [37].

Para determinar los grupos funcionales primarios del biosorbente implicados en la adsorción del cromo hexavalente, en la Fig. 2 se registraron los espectros FTIR de la cáscara de yuca antes y después de tener contacto con concentraciones previamente seleccionadas en disolución de cromo total a varias concentraciones, a saber, $15 \mathrm{mg} / \mathrm{L}$ (espectro naranja), $30 \mathrm{mg} / \mathrm{L}$ (espectro amarillo), $40 \mathrm{mg} / \mathrm{L}$ (espectro violeta), 50 $\mathrm{mg} / \mathrm{L}$ (espectro verde) y sin cromo (espectro azul). Al sobreponer todos los espectros en un mismo gráfico se encontró que hubo desplazamientos verticales, mostrando disminución del porcentaje de transmitancia cuando la concentración aumenta, además de acotamientos en ambos ejes.

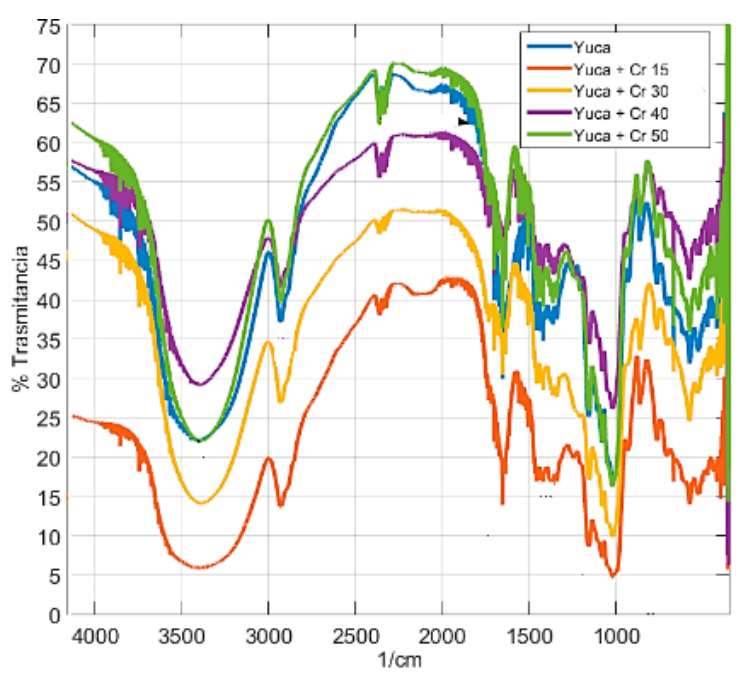

Fig. 2. Espectroscopia infrarroja con transformada de Fourier para muestras de cáscara de yuca (espectro azul), yuca $+15 \mathrm{mg} / \mathrm{L}$ de $\mathrm{Cr}$ (espectro naranja), yuca $+30 \mathrm{mg} / \mathrm{L}$ de $\mathrm{Cr}$ (espectro amarillo), yuca $+40 \mathrm{mg} / \mathrm{L}$ de $\mathrm{Cr}$ (espectro violeta) y yuca $+50 \mathrm{mg} / \mathrm{L}$ de $\mathrm{Cr}$ (espectro verde). Fuente: Autores

Los resultados muestran que la estructura química de la cáscara de yuca experimentó un cambio significativo después de la adsorción de $\mathrm{Cr}$ (VI). Las bandas de la curva antes de la adsorción a $3468 \mathrm{~cm}^{-1}$ se atribuyen al pico de absorción de vibración de estiramiento $\mathrm{O}-\mathrm{H}$ intramolecular, que es el grupo hidroxilo en la estructura lignocelulósica [38], así como a la banda de absorción característica de enlaces de hidrógeno intermoleculares entre moléculas de fenol y alcohol. Este pico se desplazó a un número de onda inferior, $3443 \mathrm{~cm}$ ${ }^{1}$, después de la adsorción de $\mathrm{Cr}$ (VI) en todos los casos y mostró una banda en el espectro, indicando que parte del O-H y los correspondientes enlaces de hidrógeno se rompieron para reaccionar con $\mathrm{Cr}$ (VI). La banda de adsorción característica a 1690 $\mathrm{cm}^{-1}$, que corresponde a la vibración de estiramiento asimétrica del enlace $\mathrm{C}=\mathrm{O}$ en ácidos carboxílicos, se debilitó significativamente después de la adsorción de Cr (VI) en todos los casos. El pico de absorción de vibración del enlace carboxilo O-H, que originalmente estaba a $1444 \mathrm{~cm}^{-1}$, se debilitó después de la adsorción de Cr (VI) y se desplazó a $1431 \mathrm{~cm}^{-1}$. Además, la banda de absorción a 874 $\mathrm{cm}^{-1}$, que representa la absorción de vibración de estiramiento del enlace $\mathrm{C}-\mathrm{H}$ aromático y fenólico, se trasladó a un número de onda inferior después de la adsorción de Cr (VI). Sobre la base de estos análisis, se concluyó que los protones ácidos de los grupos hidroxilo y carboxilo de la estructura del biosorbente se sustituyeron con $\mathrm{Cr}$ (VI) después de la adsorción. Se observaron ligeros cambios en las intensidades en las correspondientes bandas de absorción de vibraciones de los grupos funcionales reactivos. Se puede sugerir que la adsorción de $\mathrm{Cr}$ 
(VI) se basa principalmente en la adsorción química. El cromo hexavalente formó complejos con ligandos orgánicos debido a su estructura única con orbitales vacíos en la cáscara de valencia externa, como lo son $\mathrm{Cr}$ (VI) quelado con $-\mathrm{COOH}$ y - $\mathrm{OH}$ en el biosorbente. Estos resultados confirmaron que existen sitios de unión en la superficie del adsorbente que pueden acoplarse con Cr (VI). Además, las bandas de absorción se desplazan después de la adsorción de Cr (VI). Otros análisis de espectroscopia infrarroja de esta biomasa obtuvieron conclusiones semejantes [39-42].

\section{B. Análisis de adsorción en la columna}

En la Tabla 3 se muestra el diseño experimental realizado en Statgraphics Centurion ${ }^{\circledR}$ y los valores del tiempo de ruptura calculados y medidos en los 16 ensayos. En ésta se minimizó el número de variables usando el cociente que hay entre volumen del lecho con el caudal, denominado tiempo de residencia.

TABla 3. Ensayos EXPERIMENTALES y VARIABLES DE RESPUESTAS MEDIDAS.

\begin{tabular}{|c|c|c|c|}
\hline Ensayo & $\begin{array}{l}\text { Concentración } \\
\text { inicial/(mg/L) }\end{array}$ & $\begin{array}{c}\text { Tiempo de } \\
\text { residencia/min }\end{array}$ & $\begin{array}{l}\text { Tiempo de } \\
\text { ruptura/min }\end{array}$ \\
\hline 1 & 70 & 7.2 & 15 \\
\hline 2 & 100 & 4.0 & 120 \\
\hline 3 & 70 & 0.2 & 30 \\
\hline 4 & 19.5 & 3.7 & 400 \\
\hline 5 & 70 & 2.1 & 10 \\
\hline 6 & 70 & 13.3 & 90 \\
\hline 7 & 100 & 1.1 & 125 \\
\hline 8 & 120.4 & 3.7 & 30 \\
\hline 9 & 40 & 10.1 & 60 \\
\hline 10 & 40 & 2.9 & 35 \\
\hline 11 & 70 & 3.7 & 15 \\
\hline 12 & 100 & 10.1 & 40 \\
\hline 13 & 70 & 4.3 & 20 \\
\hline 14 & 40 & 4.0 & 80 \\
\hline 15 & 100 & 2.9 & 42.5 \\
\hline 16 & 40 & 1.1 & 40 \\
\hline
\end{tabular}

Fuente: Autores

A partir de los datos consignados en la Tabla 4, se realizó un análisis de varianza para determinar cuál factor tiene mayor impacto en el tiempo de ruptura.
Tabla 4. Anova multifactorial Para el tiempo De RUPtURA.

\begin{tabular}{|c|c|c|c|c|}
\hline $\begin{array}{c}\text { Fuente de } \\
\text { variación }\end{array}$ & $\begin{array}{c}\text { Grados } \\
\text { de } \\
\text { libertad }\end{array}$ & $\begin{array}{c}\text { Suma de } \\
\text { cuadrados }\end{array}$ & $\begin{array}{c}\text { Media de } \\
\text { cuadrados }\end{array}$ & $\begin{array}{c}\text { Valor } \\
\mathrm{P}\end{array}$ \\
\hline $\mathrm{A}$ & 1 & 11200 & 112100 & 0.013 \\
\hline $\mathrm{B}$ & 1 & 840 & 840 & 0.042 \\
\hline $\mathrm{AA}$ & 1 & 51100 & 51100 & 0.018 \\
\hline $\mathrm{AB}$ & 1 & 100 & 100 & 0.90 \\
\hline $\mathrm{BB}$ & 1 & 2160 & 2160 & 0.57 \\
\hline $\begin{array}{c}\text { Error } \\
\text { experimental }\end{array}$ & 10 & 63400 & 63400 & \\
\hline Total & 15 & 134000 & 13400.0 & \\
\hline
\end{tabular}

*A: Concentración inicial (mg/L); B: Tiempo de residencia (min).

\section{Fuente: Autores}

De acuerdo con el análisis de varianza, y teniendo en cuenta los valores de la suma de los cuadrados de la desviación y la prueba estadística $P$ (con un $95 \%$ de confiabilidad), se puede concluir que el factor de mayor influencia en la capacidad de remoción y el tiempo de ruptura es la concentración inicial de la solución de cromo. Con base en que la diferencia de concentración de un soluto entre una solución y un adsorbente sea la fuerza impulsora de la transferencia de masa [43] y en los resultados de otras investigaciones [44-47], no es de sorprender que la concentración del caudal de alimentación sea el factor que más afecta la variable de respuesta, hecho que el análisis de varianza confirma.

En las figuras que se analizarán en breve (Fig. 6-8) se observa un comportamiento inusual, impropio de las curvas de ruptura, consistente en que éstas no parten del origen, como regularmente lo hacen. Se realizaron múltiples ensayos con el fin de determinar si este fenómeno exhibido se debía a errores de medición; sin embargo, se determinó que no hubo tales errores en las mediciones realizadas. Esto se puede atribuir a que las concentraciones de alimentación fueron muy elevadas y que los tiempos de residencia eran muy pequeños en comparación con los de equilibrio de remoción, haciendo que el efluente comenzara a saturarse ipso facto de forma axial unidireccional, así que el lecho no era adecuado para la remoción de este metal en específico, por lo que la primera muestra tomada en $t=0$ contenía cromo en magnitudes cercanas a la mitad de la concentración inicial.

Lo anterior explica, además, los cortos tiempos de servicio de altura de lecho reportados y la dificultad de ajustar el comportamiento del tiempo de ruptura a los modelos clásicos. Este comportamiento irregular es objeto de investigación en el marco del proyecto y se adelantan estudios para identificar qué otros metales reproducen este fenómeno y establecer criterios que permitan predecir qué características de estos metales son la fuente de estas irregularidades. 


\section{REMOCIÓN DE CROMO HEXAVALENTE DE SOLUCIONES ACUOSAS USANDO CÁSCARA DE YUCA (MANIHOT ESCULENTA): EXPERIMENTOS EN COLUMNA}

\section{Efecto de la altura del lecho}

Una altura de lecho mayor significa una mayor cantidad de biosorbente, entonces es de esperar que el tiempo de servicio de la columna se prolongue con un aumento de la altura del lecho, como se puede apreciar en la Fig.3. En un lecho de menor altura predomina el efecto de dispersión axial que es la prolongación del tiempo de residencia en el flujo unidireccional debido a que no se establece un flujo tipo pistón, por otra parte, en un lecho de mayor altura tenemos más sitios activos, ya que hay mayor superficie expuesta donde se producirá la adsorción del ion metálico [48]. Se realizó una prueba a concentración inicial de cromo de $70 \mathrm{mg} / \mathrm{L}$, caudal de 7 $\mathrm{ml} / \mathrm{min}$ y un tiempo de experimento de $2400 \mathrm{~s}$ como se muestra en la Fig. 3. Es notorio que la curva con mayor altura de lecho se encuentra siempre a la derecha de las otras curvas, lo que se traduce en que a mayor altura del lecho, el tiempo de servicio del lecho es mayor.

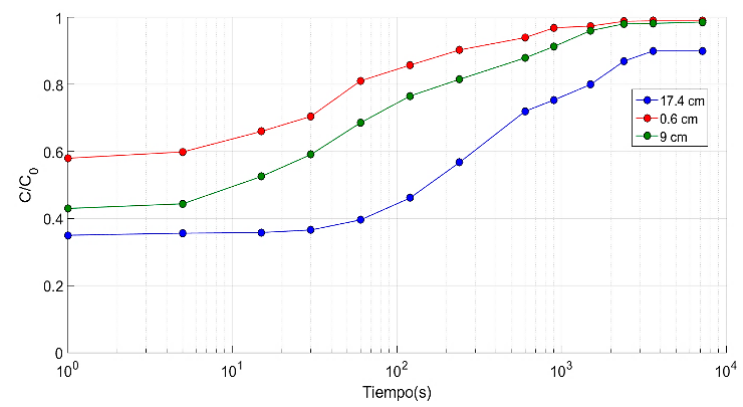

Fig. 3. Influencia de la altura del lecho en la biosorción de $\mathrm{Cr}$ sobre la cáscara de yuca $([\mathrm{Cr}]=70 \mathrm{mg} / \mathrm{L}$, caudal $=7 \mathrm{ml} / \mathrm{min}, \mathrm{t}=2400 \mathrm{~s})$. Fuente: Autores

\section{Efecto de la concentración de la alimentación}

En la Fig. 4 se logra observar un cambio de la cantidad de cromo removido a concentraciones iniciales menores. En las curvas de ruptura representadas, se exhibió una importante diferencia del tiempo de ruptura entre las curvas cuyas concentraciones iniciales fueron de 70, 19.54 y $120.4 \mathrm{mg} / \mathrm{L}$. Para la curva de $120.4 \mathrm{mg} / \mathrm{L}$, el lecho se saturó en un tiempo menor por ser mayor la concentración. Como bien es conocido de los fenómenos de adsorción, la fuerza impulsora del transporte de materia es la diferencia de concentración entre la disolución que contiene el adsorbato y el adsorbente, y que el coeficiente de difusión toma menores valores a diferencias de concentración menores, ocasionando una transferencia más lenta, en nuestro caso, del cromo en disolución hacia los sitios activos de la superficie donde se hace efectivo el transporte de masa.
De forma contraria, un aumento en la concentración en la corriente de alimentación provoca un aumento en la diferencia de concentración traduciéndose en un mayor valor del coeficiente de transferencia de masa o, equivalentemente, en un proceso de difusión de metal más rápido [43].

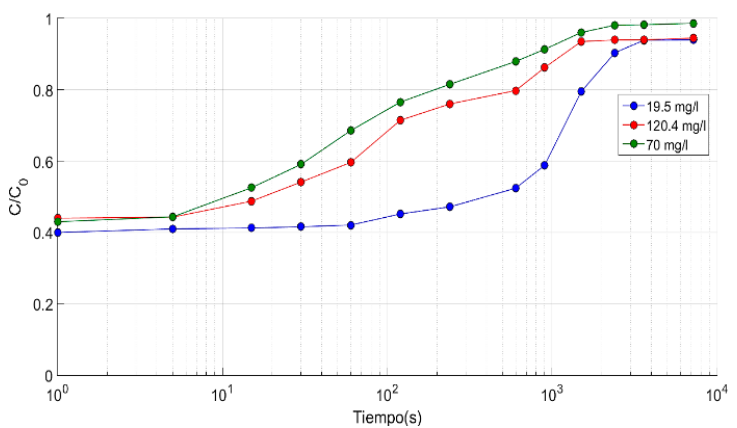

Fig. 4. Influencia de la concentración de alimentación en la biosorción de Cr sobre la cáscara de yuca (caudal $=7 \mathrm{ml} / \mathrm{min}, \mathrm{L}=9 \mathrm{~cm}, \mathrm{t}=2400 \mathrm{~s}$ ). Fuente: Autores

\section{E. Efecto del caudal de alimentación}

A partir de la Fig. 5 se puede analizar el efecto que el caudal tiene sobre la remoción del ion metálico. A medida que el flujo de alimento es mayor, se observa concretamente una importante disminución del tiempo de ruptura.

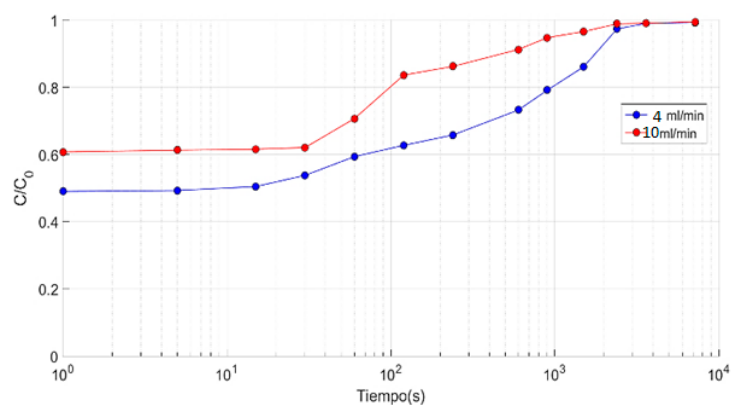

Fig. 5. Influencia del caudal de alimentación en la biosorción de $\mathrm{Cr}$ total sobre la cáscara de yuca $([\mathrm{Cr}$ total $]=40 \mathrm{mg} / \mathrm{L}, \mathrm{L}=14 \mathrm{~cm}, \mathrm{t}=2400 \mathrm{~s})$. Fuente: Autores

Para el análisis de la influencia del caudal de alimentación del lecho, se realizó un ensayo donde se mantuvo constante la concentración de cromo en 40 $\mathrm{mg} / \mathrm{L}$ y altura del lecho en $14 \mathrm{~cm}$, mientras se usaron dos valores de caudal de 10 y $4 \mathrm{~mL} / \mathrm{min}$. En todo momento la curva de $4 \mathrm{~mL} / \mathrm{min}$ se mantuvo más a la izquierda de la de $10 \mathrm{~mL} / \mathrm{min}$, mostrando así que a mayor caudal, el tiempo de saturación del lecho es mucho más prolongado. La diferencia radica en que a medida que el caudal entra en contacto con el lecho y pasa a través de él, se va saturando la 
parte del lecho por el cual ha ascendido la película de fluido, y debajo de la película del fluido se tendrá una concentración de cromo mayor a la parte superior de la película teniendo en cuenta que a medida que avanza el fluido en el lecho, hay caídas de presión por la resistencia al flujo y porosidad dentro del lecho ocupada por aire. En caso de que el flujo sea lo suficientemente alto como aliviar las caídas de presión, tiene lugar el retromezclado de la película superior con la que se encuentra por debajo de ésta, mientras se tenga un flujo en el que, aun teniendo caídas de presión dentro del lecho, no se verá afectado el ascenso del fluido, marcando un ritmo de ascenso casi constante. Se puede explicar este comportamiento entendiendo que cuando se aumenta el caudal de alimentación se favorece la interacción entre las moléculas de cromo con el medio adsorbente, lo cual tiene una gran importancia sobre la velocidad a la cual se produce la transferencia de materia [49]. Además, un aumento en el caudal incorpora consigo un menor tiempo de residencia, que es sinónimo de una minimización en el tiempo de contacto entre el adsorbente y el adsorbato.

\section{F. Ajuste a modelos}

1) Modelo de superficie de respuesta. El modelo matemático indica la relación existente entre el tiempo de ruptura y las principales fuentes de variación, que para este caso son el tiempo de residencia que se comprende como la relación entre el caudal y la altura del lecho, y, por otro lado, la concentración inicial del cromo en la solución que entra en la columna empacada.

$$
\begin{aligned}
& \text { Tiempo de ruptura }=0.1759+1.147 x \\
& -0.0647 y+0.9468 x^{2}-0.3909 x y+0.00657 y^{2}- \\
& 0.9687 x^{3}-0.2866 x^{2} y+0.1575 x y^{2}+0.1013 y^{3}(17)
\end{aligned}
$$

Donde $x$ es la concentración inicial e $y$ el tiempo de residencia en cada caso. El valor de $R^{2}$ fue 0.9456 para el tiempo de ruptura. La superficie de respuesta elaborada se muestra en la Fig. 6.

En la Fig. 6 se puede observar que el tiempo de ruptura disminuye con un aumento de la concentración inicial de cromo, pero a medida que aumenta la concentración inicial se notan variaciones irregulares con respecto al tiempo de ruptura, las cuales están relacionadas con intervalos particulares de caudal y altura del lecho que aplican sus efectos de manera implícita en el tiempo de residencia, ya que fue calculado a partir de las mismas. Ejemplo de lo anterior se puede observar en el plano de intersección de tiempo de residencia igual a dos minutos, donde se notan al menos tres cambios en la monotonía de la curva de intersección con el plano antes mencionado a lo largo de todo el intervalo de concentración inicial.
Disponer de una relación matemática para poder predecir valores para una variable en concreto es, desde luego, una herramienta valiosa. De esta forma, el modelo de superficie de respuesta proporciona una expresión simple al ser un polinomio en el cual se pueden encontrar sus puntos máximos mediante sencillas diferenciaciones parciales. Así, el modelo de superficie de respuesta entrega dos importantes funciones: en primer lugar, estimar cualquier valor de tiempo de ruptura para cualquier combinación de los factores en el dominio seleccionado y, en segundo lugar, hallar la mejor combinación de factores, la que alcanza el máximo tiempo de ruptura mediante diferenciación parcial.

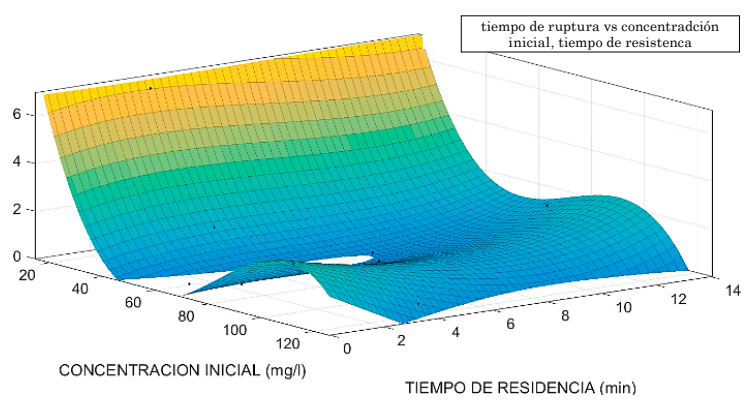

Fig. 6. Superficie de respuesta para el tiempo de ruptura en función de la concentración inicial y el tiempo de residencia. Fuente: Autores

Derivando parcialmente la ecuación (17) se obtiene el tiempo de ruptura óptimo solucionando el sistema $2 \times 2$ resultante:

$$
\frac{\partial T_{\text {rup }}}{\partial x}=0 \quad y \quad \frac{\partial T_{\text {rup }}}{\partial y}=0
$$

De donde

$$
x=0.8306 \frac{\mathrm{mg}}{\mathrm{L}} \quad \text { e } \quad y=1.00 \mathrm{~min}
$$

Reemplazando estos valores en la ecuación (17), se tiene que el tiempo de ruptura óptimo es de 0.88 min. Se hace referencia como óptimo a este valor porque es el que usa menos volumen de lecho y alcanza la capacidad de adsorción máxima.

2) Modelo de Yoon-Nelson. Este modelo en algunos casos presenta aproximaciones aceptables, pero en general no es lo suficientemente acertado; por ejemplo, en la Fig. 7 se muestra lo más acercado que pudo estar el modelo de la curva de ruptura experimental con un valor de $\mathrm{R}^{2}$ de 0.9226 , en el cual se pudo apreciar que solamente se intersectan las dos curvas cuando están llegando a la saturación completa del lecho, no obstante, en los puntos anteriores a estos se aprecian diferencias significativas, por lo que se concluye que este modelo no ajustó adecuadamente los datos experimentales. 


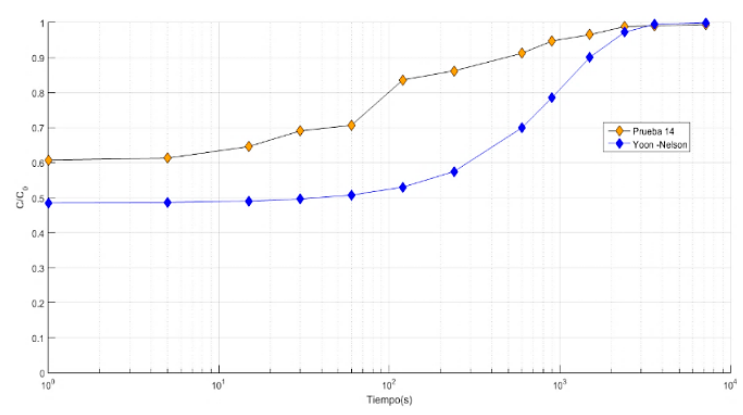

Fig. 7. Comparación entre la curva de ruptura experimental de la prueba 14 y la modelación de Yoon- Nelson. Fuente: Autores

3) Modelo de dosis y respuesta. Para este modelo solo unos de los pocos resultados obtenidos logran superar un $\mathrm{R}^{2}>0.9$, el cual es el criterio de aceptabilidad para los ajustes obtenidos para cada curva de ruptura elaborada. Solo para las pruebas 2 , $3,4,10$, y 14 se obtuvieron resultados aceptables, como se muestra en la figura 8 , lo cual confirma un acercamiento más cerrado del modelo a estas curvas. Éste, en general, muestra un mejor ajuste entre los modelos clásicos usados, pero para algunos casos, cuando se tienen condiciones de operación específicas, el modelo no permite reproducir el comportamiento de la columna, debido a que las constantes obtenidas no guardan concordancia física, por ejemplo, tiempos negativos, haciendo que el escalamiento mediante este modelo no sea una opción atractiva.

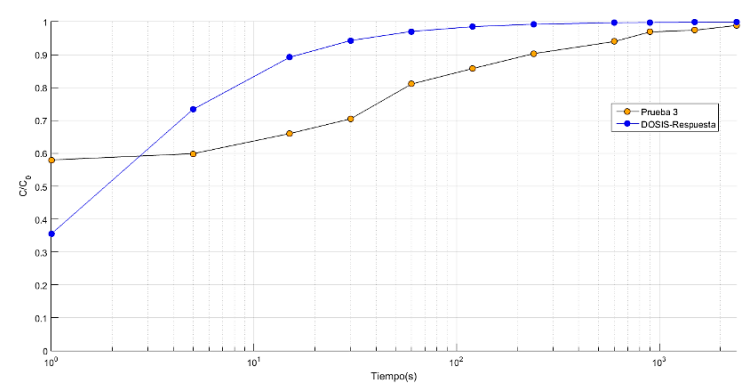

Fig. 8. Comparación entre la curva de ruptura experimental de la prueba $3, \mathrm{~L}=0.6 \mathrm{~cm}, \mathrm{Q}=7 \mathrm{ml} / \mathrm{min}$, $\mathrm{C} 0=70 \mathrm{mg} / \mathrm{L}, \mathrm{pH}=3 \mathrm{con}$ el predicho por el modelo de dosis y respuesta para las mismas condiciones. Fuente: Autores

\section{Conclusiones}

Se preparó un montaje que permitió alcanzar un proceso semicontinuo para la adsorción de cromo hexavalente sobre cáscara de yuca. Se obtuvieron resultados esperados: un aumento en el caudal satura el lecho más rápido debido a que hay una transferencia más rápida de cromo hacia los sitios activos de adsorción que aprovechan el fenómeno de solvatación, hecho que es deducido del análisis in- frarrojo que reveló la presencia de grupos hidroxilos y carboxilos que implican la quelación del cromo en el biosorbente, de lo cual se puede afirmar que la adsorción es de naturaleza química, predominando un fenómeno de saturación axial unidireccional. Una altura de lecho mayor se traduce en un aumento del volumen de éste y, por tanto, también de sitios de transferencia que son aprovechados para atraer al ion metálico y hacen que el tiempo de servicio de la columna sea mayor, es decir, el tiempo de saturación aumenta. Por último, un aumento en la concentración del metal en el caudal de alimentación satura a mayor velocidad el lecho debido a que la fuerza impulsora de la transferencia de masa es precisamente la diferencia de concentraciones del ion entre la disolución y el biosorbente; en efecto, la concentración inicial del alimento es el factor que tiene mayor efecto sobre la capacidad de remoción y el tiempo de ruptura, hecho que fue confirmado estadísticamente en el análisis de varianza. Si bien se observó una disminución de la concentración de cromo a la salida del lecho empacado, la columna con este material se satura muy rápidamente y el equilibrio del sistema no permite una remoción que muestre potencialidades de aplicación industrial de este material para la remoción del cromo.

Respecto a la caracterización de la cáscara de yuca, ésta se identificó como un biosorbente ácido con $\mathrm{pH}$ de 4.5, un diámetro de partícula en el rango de 250-500 $\mathrm{\mu m}$, una densidad de $0.41 \mathrm{~g} / \mathrm{ml}$, una porosidad de $59.2 \%$ y una humedad promedio de $10.76 \%$, características inherentes de este tipo de biomasa.

Se apreció que el diseño experimental fue adecuado para ajustar el comportamiento del tiempo de ruptura al modelo estadístico de superficie de respuesta, el cual es una potente herramienta que permite estimar tiempos de ruptura en los rangos considerados y a través del cual se calculó el tiempo de ruptura óptimo.

\section{Referencias}

[1] J. M. Chen y O. J. Hao, "Microbial chromium (VI) reduction," Critical Reviews in Environmental Science and Technology, vol. 28, pp. 219-251, 1998.

[2] A. Papandreou, C. Stournaras y D. Panias, "Copper and cadmium adsorption on pellets made from fired coal fly ash," Journal of Hazardous Materials, vol. 148, pp. 538547, 2007.

[3] Y. Sharma, "Economic treatment of cadmium (II)-rich hazardous waste by indigenous material," Journal of colloid and interface science, vol. 173, pp. 66-70, 1995.

[4] K. Singh, A. Singh y S. Hasan, "Low cost bio-sorbent 'wheat bran'for the removal of cadmium from wastewater: kinetic and equilibrium studies," Bioresource technology, vol. 97, pp. 994-1001, 2006.

[5] WHO, Environmental Health Criteria 221: Zinc vol. 11. Geneva, 2001.

[6] D. Manohar, K. A. Krishnan y T. Anirudhan, "Removal of mercury (II) from aqueous solutions and chlor-alkali industry wastewater using 2-mercaptobenzimidazoleclay," Water Research, vol. 36, pp. 1609-1619, 2002. 
[7] A. Öztürk, "Removal of nickel from aqueous solution by the bacterium Bacillus thuringiensis," Journal of Hazardous Materials, vol. 147, pp. 518-523, 2007.

[8] F. Granados-Correa y J. Serrano-Gómez, "CrO4 2- ions adsorption by Fe-modified pozzolane," Separation Science and Technology, vol. 44, pp. 924-936, 2009.

[9] J. Wang y C. Chen, "Biosorbents for heavy metals removal and their future," Biotechnology advances, vol. 27, pp. 195-226, 2009

[10] S. H. Abbas, I. M. Ismail, T. M. Mostafa y A. H. Sulaymon, "Biosorption of heavy metals: a review," Journal of Chemical Science and Technology, vol. 3, pp. 74-102, 2014.

[11] M. Owlad, M. K. Aroua, W. A. W. Daud, S. Baroutian, "Removal of Hexavalent Chromium-Contaminated Water and Wastewater: A Review," Water Air and Soil Pollution, vol. 200(1), pp. 59-77, 2008.

[12] N. H. Shaidan, U. Eldemerdash y S. Awad, "Removal of $\mathrm{Ni}$ (II) ions from aqueous solutions using fixed-bed ion exchange column technique," Journal of the Taiwan Institute of Chemical Engineers, vol. 43, pp. 40-45, 2012.

[13] A. Mittal, J. Mittal, A. Malviya y V. Gupta, "Removal and recovery of Chrysoidine $\mathrm{Y}$ from aqueous solutions by waste materials," Journal of Colloid and Interface Science, vol. 344, pp. 497-507, 2010.

[14] L. Altaş y H. Büyükgüngör, "Sulfide removal in petroleum refinery wastewater by chemical precipitation," Journal of hazardous materials, vol. 153, pp. 462-469, 2008.

[15] V. Gupta, R. Jain, A. Mittal, M. Mathur y S. Sikarwar, "Photochemical degradation of the hazardous dye Safranin-T using TiO 2 catalyst," Journal of colloid and interface science, vol. 309, pp. 464-469, 2007.

[16] R.-S. Juang y R.-C. Shiau, "Metal removal from aqueous solutions using chitosan-enhanced membrane filtration," Journal of Membrane Science, vol. 165, pp. 159167, 2000.

[17] V. K. Gupta, R. Jain y S. Varshney, "Electrochemical removal of the hazardous dye Reactofix Red 3 BFN from industrial effluents," Journal of Colloid and Interface Science, vol. 312, pp. 292-296, 2007.

[18] A. Mittal, L. Kurup y V. K. Gupta, "Use of waste materials-bottom ash and de-oiled soya, as potential adsorbents for the removal of amaranth from aqueous solutions," Journal of Hazardous Materials, vol. 117, pp. 171-178, 2005.

[19] N. Ahalya, T. Ramachandra y R. Kanamadi, "Biosorption of heavy metals," Res. J. Chem. Environ, vol. 7, pp. 71-79, 2003.

[20] Z.-n. Huang, X.-l. Wang y D.-s. Yang, "Adsorption of Cr (VI) in wastewater using magnetic multi-wall carbon nanotubes," Water Science and Engineering, vol. 8, pp. 226-232, 2015.

[21] Y. Sharma, V. Srivastava, C. Weng y S. Upadhyay, "Removal of $\mathrm{Cr}$ (VI) from wastewater by adsorption on iron nanoparticles," The Canadian Journal of Chemical Engineering, vol. 87, pp. 921-929, 2009.

[22] B. Volesky, "Biosorption and biosorbents," in Biosorption of heavy metals. vol. I, ed Florida, Boca raton: CRC Press, p. 4, 1990.

[23] D. Schwantes, A. C. Gonçalves Jr., G. F. Coelho, M. A. Campagnolo, D. C. Dragunski, C. R. T. Tarley, A. J. Miola y E. A. Volz Leismann, "Chemical Modifications of Cassava Peel as Adsorbent Material for Metals Ions from Wastewater," Journal of Chemistry, vol. 2016, pp. $1-15,2016$.

[24] S. Shukla y V. Skhardande, "Column studies on metal ion removal by dyed cellulosic materials," Journal of Applied Polymer Science, vol. 44, pp. 903-910, 1992.

[25] M. Calero, G. Blázquez, F. Hernáinz, A. Ronda y M. A. Martín-Lara "Biosorción de cobre con corteza de pino en columna de lecho de fijo: optimización de las variables del proceso," Afinidad LXIX, vol. 559, pp. 175-184, 2012.
[26] O. Freitas, C. Delerue-Matos y R. Boaventura, "Optimization of $\mathrm{Cu}$ (II) biosorption onto Ascophyllum nodosum by factorial design methodology," Journal of Hazardous Materials, vol. 167, pp. 449-454, 2009.

[27] S. Hasan, P. Srivastava y M. Talat, "Biosorption of lead using immobilized Aeromonas hydrophila biomass in up flow column system: Factorial design for process optimization," Journal of Hazardous Materials, vol. 177 (1-3), pp. 312-322, 2010.

[28] X. Jing, Y. Cao, X. Zhang, D. Wang, X. Wu y H. Xu, "Biosorption of $\mathrm{Cr}$ (VI) from simulated wastewater using a cationic surfactant modified spent mushroom," Desalination, vol. 269 (1-3), pp. 120-127, 2011.

[29] D. Park, Y. Yun, D. Lee y J. Park, "Optimum condition for the removal of $\mathrm{Cr}$ (VI) or total $\mathrm{Cr}$ using dried leaves of Pinus densiflora," Desalination, vol. 271 (1-3), pp. 309 $314,2011$.

[30] D. Ranjan y S. Hasan, "Parametric Optimization of Selenite and Selenate Biosorption Using Wheat Bran in Batch and Continuous Mode," J. Chem. Eng. Data, vol. 55(11), pp. 4808-4816, 2010.

[31] Y. Safa y H. Bhatti, "Adsorptive removal of direct textile dyes by low cost agricultural waste: application of factorial design analysis," Chemical Engineering Journal, vol. 167, pp. 35-41, 2011.

[32] Métodos de Ensayo: Determinación del pH de la muestra, ICONTEC, 1998.

[33] M. Carrier, A. Loppinet-Serani, D. Denux, J.-M. Lasnier, F. Ham-Pichavant, F. Cansell y C. Aymonier, "Thermogravimetric analysis as a new method to determine the lignocellulosic composition of biomass," Biomass and Bioenergy, vol. 35, pp. 298-307, 2011.

[34] K. Vijayaraghavan y Y. Yun, "Chemical modification of Corynebacterium glutamicum to improve methylene blue biosorption," Chemical Engineering Journal, vol. 145, pp. $1-6,2008$.

[35] L. Lopez, A. Acosta y R. Gelves, "Evaluación de pretratamientos químicos para la hidrólisis enzimática de residuos lignocelulósicos de yuca (Manihot esculenta Crantz)," Rev. Fac. Ing. Univ. Antioquia, vol. 69, p. 4, 2013.

[36] P. Wilaipon, "Density Equation of Cassava-Stalk Briquettes Under Moderate Die-Pressure," American Journal of Applied Sciences, vol. 7(5), pp. 698-701, 2010.

[37] J. A. García y E. E. Yañez, "Generación y uso de biomasa en plantas de beneficio de palma de aceite en Colombia," Revista Palmas, vol. 31, pp. 41-48, 2010.

[38] M. C. Sifontes y M. E. Domine, "Lignina, estructura y aplicaciones: métodos de despolimerización para la obtención de derivados aromáticos de interés industrial," Avances en Ciencias e Ingeniería, vol. 4, pp. 15-46, 2013.

[39] H. A. Acosta Arguello, C. A. Barraza Yance y A. R. Albis Arrieta, "Adsorción de cromo (VI) utilizando cáscara de yuca (Manihot esculenta) como biosorbente: Estudio cinético," Ingeniería y Desarrollo, vol. 35, 2017.

[40] A. R. Albis, L. V. Cajar y M. I. Domínguez, "Análisis cinético de la adsorción de $\mathrm{Cr}$ (VI) en soluciones acuosas a concentraciones de 10-20 mg/L con el uso de cáscara de yuca amarga (Manihot esculenta)," Prospectiva, vol. 13, pp. 64-71, 2015.

[41] A. Kurniawan, A. N. Kosasih, J. Febrianto, Y.-H. Ju, J. Sunarso, N. Indraswati, S. Ismadji, "Evaluation of cassava peel waste as lowcost biosorbent for Ni-sorption: Equilibrium, kinetics, thermodynamics and mechanism," Chemical engineering journal, vol. 172, pp. 158-166, 2011.

[42] A. N. Kosasih, J. Febrianto, J. Sunarso, Y.-H. Ju, N. Indraswati y S. Ismadji, "Sequestering of $\mathrm{Cu}$ (II) from aqueous solution using cassava peel (Manihot esculenta)," Journal of hazardous materials, vol. 180, pp. 366$374,2010$.

[43] R. E. Treybal, A. García Rodríguez, and F. J. Lozano, Operaciones de tranferencia de masa. McGraw-Hill, 1988. 


\section{REMOCIÓN DE CROMO HEXAVALENTE DE SOLUCIONES ACUOSAS USANDO CÁSCARA DE YUCA (MANIHOT ESCULENTA): EXPERIMENTOS EN COLUMNA}

[44] J. V. Ríos y G. C. Ortega, "Predicción de las curvas de ruptura para la remoción de plomo (II) en disolución acuosa sobre carbón activado en una columna empacada," Revista Facultad de Ingeniería, pp. 141-158, 2013.

[45] E. Tomczak, "Water purification from heavy metal ions in a packed column," Separation Science and Technology, vol. 48, pp. 2270-2276, 2013

[46] Ö. Saatçilar, N. Şatiroğlu, S. Bektaş, Ö. Genç y A. Denizli, "Packed-bed columns with dye-affinity microbeads for removal of heavy metal ions from aquatic systems," Reactive and Functional Polymers, vol. 50, pp. 41-48, 2002 .
[47] S. Mohan y G. Sreelakshmi, "Fixed bed column study for heavy metal removal using phosphate treated rice husk," Journal of Hazardous Materials, vol. 153, pp. 7582, 2008.

[48] E. Gallardo, S. Maioco y N.Francois, "Remoción de un colorante básico por adsorción con Quitosano y con un material compuesto de Quitosano-Zeolita," 2008.

[49] A. García-Abuín, D. Gómez-Díaz, J. M. Navaza, and I. Vidal-Tato, "Comportamiento hidrodinámico y absorción de dióxido de carbono mediante reacción química con glucosamina en un reactor Air-Lift" Av. en Ciencias e Ing., vol. 1, no. 2, pp. 25-36, 2010. 